\title{
Substitutions in the Capsids of Poliovirus Mutants Selected in Human Neuroblastoma Cells Confer on the Mahoney Type 1 Strain a Phenotype Neurovirulent in Mice
}

\author{
THÉRÈSE COUDERC ${ }^{1}$ NICOLE GUÉDO,${ }^{1}$ VINCENT CALVEZ, ${ }^{1}$ ISABELLE PELLETIER, ${ }^{1}$ \\ JAMES HOGLE, ${ }^{2}$ FLORENCE COLBËRE-GARAPIN, ${ }^{1}$ AND BRUNO BLONDEL ${ }^{1 *}$ \\ Unité de Virologie Médicale, Institut Pasteur, 75724 Paris cedex 15, France, ${ }^{1}$ and Department of Biological \\ Chemistry and Molecular Pharmacology, Harvard Medical School, Boston, Massachussets $02115^{2}$
}

Received 17 May 1994/Accepted 26 August 1994

\begin{abstract}
Poliovirus (PV) type 1 mutants selected in human neuroblastoma cells persistently infected (PVpi) with the wild-type Mahoney strain exhibited a mouse-neurovirulent phenotype. Four of the five substitutions present in the capsid proteins of a PVpi were demonstrated to extend the host range of the Mahoney strain to mice. These new mouse-neurovirulent determinants were located in the three-dimensional structure of the viral capsid; two of them (residues 142 of VP2 and 60 of VP3) were located in loops exposed at the surface of the protein shell, whereas the other two (residues 43 of VP1 and 62 of VP4) were located on the inside of the capsid. VP1 residue 43 and VP2 residue 142 substitutions were also selected in a PVpi derived from the attenuated Sabin strain. We suggest that the selective pressure of human neuroblastoma cell factor(s) involved in early steps of PV multiplication could be responsible for the arising of amino acid substitutions which confer adaptation to the mouse central nervous system to $\mathbf{P V}$.
\end{abstract}

Poliovirus (PV) is a human enterovirus of the Picornaviridae family and is classified into three serotypes (PV-1, PV-2, and PV-3). The PV virion consists of a single-stranded RNA molecule of positive polarity surrounded by an icosahedral capsid made of 60 copies of each of the four structural polypeptides VP1, VP2, VP3, and VP4. The precise structure of the capsid proteins in infectious PV has been established by $\mathrm{X}$-ray crystallography $(7,10)$. The human PV receptor has been identified as a member of the immunoglobulin superfamily of proteins $(12,17)$, and recently it has been shown that the lymphocyte homing receptor CD44 may be involved in the interactions between PV and its receptor (23).

$\mathrm{PV}$ is the causative agent of poliomyelitis, an acute disease of the central nervous system of humans that in some cases results in paralysis. Wild-type strains of PV generally exhibit a high neuropathogenic potential, whereas Sabin vaccine strains of PV are attenuated in their ability to infect the central nervous system. Although most wild-type PV strains can infect only primates, the Lansing strain of PV-2 has been adapted to mice, in which it causes paralytic disease when inoculated intracerebrally. In contrast, many other PV strains, including the type 1 Mahoney strain, although highly neurovirulent in primates, are avirulent in mice. The genetic basis for host restriction of PV-1 in mice was first studied by construction of viral recombinants between PV-1 and PV-2 strains (14). This study revealed that the capsid-encoding region of PV-2/Lansing is sufficient to confer the mouse-virulent phenotype to PV-1/Mahoney. A more accurate location of mouse adaptation determinants of PV-1 has been obtained by manipulating cDNA of PV-1/Mahoney $(16,18,19)$ and by isolation in vivo and characterization of mutants after passage of the virus in the mouse central nervous system (5). These determinants can be classified into two groups: (i) determinants localized at the surface of the protein shell in the amino acid sequence from 94

* Corresponding author. Mailing address: Unité de Virologie Médicale, Institut Pasteur, 28 Rue du Docteur Roux, 75724 Paris cedex 15, France. Phone: (33).1.45.68.87.62. Fax: (33).1.45.68.87.80. to 102 of the B-C loop of VP1, a major component of the antigenic neutralization site 1 (10); and (ii) determinants located inside the viral capsid corresponding to residues at positions 22, 40, and 54 of VP1 and 31 of VP2 $(7,10)$. It seems likely that PV strains which cannot infect mice are blocked in one or more of the early steps of mouse nerve cell infection, since transgenic mice expressing the human PV receptor are susceptible to infection with all three serotypes of $\operatorname{PV}(13,22)$. The mutations in the first group (located on the outside surface of the virus) may act directly by affecting interactions with specific receptors within the mouse central nervous system. Alternatively, these mutations as well as the mutations in the second group (located on the inside of the virus) may play a role in controlling receptor-mediated conformational changes, i.e., the extrusion of the N terminus of VP1 and the release of VP4 $(8,9,15)$, and thus allow PV to overcome the host range restriction $(5,18)$.

Although PV is usually considered to be a lytic virus, it has been shown that virus from all three serotypes of PV can induce a persistent infection in human neuroblastoma cells such as IMR-32 cells (4). The PV mutants recovered from neuroblastoma cells persistently infected (PVpi) with the Sabin strains exhibited phenotypic properties which differed greatly from those of the parental strains (21). In particular, the PVpi titers were 1 to $5 \log _{10}$ units higher in IMR-32 cells than on nonneuronal HEp-2c cells, while parental viruses had similar titers on both lines. In correlation with this modified cell specificity, PVpi could establish secondary persistent infections in HEp-2c cells. Mutations responsible for this phenotype were located in a genomic region, between nucleotides 1148 and 3481 , of a PVpi derived from the attenuated PV-1/Sabin strain (PVpiS11) (3). This region carried 11 missense mutations mapping in the genes of capsid proteins VP1 and VP2 (2). Surprisingly, all three independent PVpi selected in IMR-32 persistently infected with the attenuated PV-1/Sabin strain exhibited a point mutation $(\mathrm{C} \rightarrow \mathrm{U})$ at nucleotide 2544 inducing amino acid change of Thr-22 of VP1 to Met. Since substitution $\mathrm{Thr} \rightarrow$ Ile at this same position renders PV-1/Mahoney neuro- 


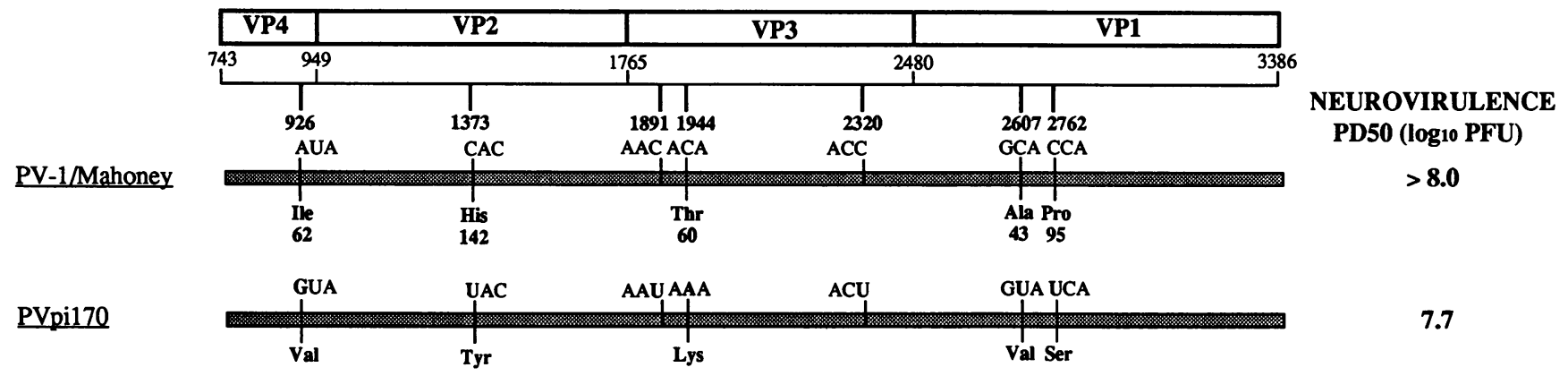

FIG. 1. Genomic organization of the region of the PV-1/Mahoney genome encoding the capsid proteins. The positions of mutated nucleotides and substituted amino acids are indicated in boldface. $\mathrm{PD}_{50} \mathrm{~s}$ for mice are shown at the right.

virulent for mice (5), we first investigated whether substitution Thr-22 $\rightarrow$ Met in VP1 can confer the mouse-neurovirulent phenotype to the mouse-avirulent PV-1/Mahoney. As differences between PV-1/Mahoney and PV-1/Sabin genomes include a silent mutation at nucleotide $2545(A \rightarrow G)$, we had to change two nucleotides $(\mathrm{C}-2544 \rightarrow \mathrm{U}$ and $\mathrm{A}-2545 \rightarrow \mathrm{G})$ to introduce the Thr-22 $\rightarrow$ Met change in polypeptide VP1 of PV-1/ Mahoney. Both mutations were introduced in the VP1 gene by site-directed mutagenesis. The PV-1/Mahoney cDNA sequence from nucleotide 2243 to 3064 (PstI-KpnI fragment) was inserted into the polylinker of M13mp18 vector DNA (Amersham), resulting in M13/KK-VP1. The mutations were introduced in the single-strand form of M13/KK-VP1, using the oligonucleotide primer (5'-CGGCAATGTCTAGAGACG C- $\left.3^{\prime}\right)$ and the oligonucleotide-directed in vitro mutagenesis system (Sculptor; Amersham) in accordance with the manufacturer's instructions. The 484-bp NheI-SnaBI fragment from the mutated replicative form was used to substitute for the corresponding fragment in pKK17 (11), containing the fulllength PV-1/Mahoney cDNA. The resulting plasmid, pKKVP1 $1 M_{22}$, was purified and then used to transfect IMR-32 cells as previously described (5). Upon recovery of the corresponding virus, Mah-KKVP1M ${ }_{22}$, the sequence of the viral genome was verified in the mutated region and shown to carry the expected mutations. Mah-KKVP1M ${ }_{22}$ was tested for its neurovirulent phenotype in mice. Groups of six 21-day-old female OF1 mice (IFFA-CREDO) were inoculated intracerebrally with $0.03 \mathrm{ml}$ of serial dilutions of viral stock, and the animals were observed daily for 21 days to score paralysis or death. The mutated virus was neurovirulent for mice, and the dose which paralyzed $50 \%$ of the animals $\left(\mathrm{PD}_{50}\right)$ was $10^{8} \mathrm{PFU}$. The sequence of viral RNA recovered from the spinal cord of one paralyzed mouse was verified as described previously (5) and shown to carry the mutations. Thus, the $\mathrm{Thr} \rightarrow$ Met substitution at residue 22 of VP1 resulted in a mouse-neurovirulent PV-1/ Mahoney, indicating that mutations able to extend the host range of PV-1 to mice could be selected during the persistent infection of human neuroblastoma cells.

To investigate whether this system could be used as a new approach to select mouse-adapted mutants, we attempted to isolate mouse-virulent mutants derived from IMR-32 cells persistently infected with PV-1/Mahoney. In PV-1/Sabin, attenuating mutations outside the capsid region preclude screening for neurovirulence in the mouse. In contrast, the wild-type PV-1/Mahoney strain is fully virulent, and PVpi mutants derived from the Mahoney strain can thus be screened directly for mouse adaptation. IMR-32 cells were inoculated with PV-1/Mahoney at a multiplicity of infection (MOI) of either 1 or $10050 \%$ tissue culture infectious doses per cell to establish a persistent infection as previously described (4). Two and a half months after the establishment of the persistent infection, PVpi mutants were isolated and cloned by plaque purification onto IMR-32 cells. Two clones of PVpi mutants for each MOI (PVpi170 and PVpi172 for an MOI of 100; PVpi175 and PVpi177 for an MOI of 1) were tested for their neurovirulent phenotypes in mice. The neurovirulence test was performed by injecting $10^{8}$ PFU of each mutant into mice. Fifteen of 28,12 of 28,3 of 22 , and 8 of 22 mice inoculated with PVpi170, PVpi172, PVpi175, and PVpi177, respectively, developed paralysis. These results revealed that the all four PVpi mutants isolated from human neuroblastoma cells were able to infect the mouse central nervous system, in contrast to the parental strain PV-1/Mahoney. We retained the most mouse-neurovirulent PVpi mutant, PVpi170, for further analysis.

We estimated the degree of neurovirulence of PVpi170 in mice by determining the $\mathrm{PD}_{50}$ as described above. The $\mathrm{PD}_{50}$ value was $10^{7.7}$ PFU, whereas inoculation of $10^{8}$ PFU of PV-1/Mahoney induced no paralysis in mice.

To investigate the genetic basis of adaptation to mice of PVpi170, we looked for mutations in the capsid proteinencoding region $\mathrm{P} 1$ of the PVpi170 genome. To ensure that mutations did not arise during virus growth in the mouse central nervous system, the sequence was first determined for the genome of virus recovered from the spinal cord of one paralyzed mouse inoculated with PVpi170 (PVpi170sp), and all nucleotide changes found were then checked in the PVpi170 genome. Total cytoplasmic RNA from PVpi170spand PVpi170-infected IMR-32 cells was used for sequencing as described previously (6). Seven point mutations were identified in the P1 region of PVpi170sp genome and were also detected in the PVpi170 genome (Fig. 1). Therefore, all of the mutations were selected during the persistent infection of IMR-32 cells with PV-1/Mahoney. Five of them, i.e., Ala-43 $\rightarrow$ Val and Pro-95 $\rightarrow$ Ser in VP1, His-142 $\rightarrow$ Tyr in VP2, Thr-60 $\rightarrow$ Lys in VP3, and Ile-62 $\rightarrow$ Val in VP4, were missense mutations.

To determine whether the same missense mutations would be selected in other PVpi derived from the Mahoney strain, we looked for these mutations in the genomes of PVpi172, PVpi175, and PVpi177. The mutations modifying amino acid 95 of VP1, 142 of VP2, and 60 of VP3 were also found in the genomes of the three viruses. The mutation modifying amino acid 43 of VP1 was found in the genome of PVpi172 but not in that of PVpi175 or -177 . The mutation modifying amino acid 62 of VP4 was found in PVpi170 only. By comparing the region encoding the capsid proteins of PVpi170 with that of PVpiS11, derived from the attenuated PV-1/Sabin strain $(2,21)$, it appeared that four mutations of PVpi170 (at nucleotides 2607, 2672,1373 , and 1944) created common residues at homolo- 
TABLE 1. Neurovirulence of PV-1/Mahoney mutants carrying individual mutations identified in the protein-encoding region of the

PVpi170 genome

\begin{tabular}{|c|c|c|c|c|c|c|}
\hline \multirow{2}{*}{ Virus } & \multicolumn{2}{|c|}{ Modified nucleotide } & \multicolumn{3}{|c|}{ Modified amino acid } & \multirow{2}{*}{$\begin{array}{c}\mathrm{PD}_{50} \\
\left(\log _{10} \mathrm{PFU}\right)\end{array}$} \\
\hline & Position & Nucleotide & Protein & Position & Residue & \\
\hline Mah-KKVP1V & 2607 & $\mathbf{U}$ & VP1 & 43 & Val & 6.0 \\
\hline Mah-KKVP1S $_{95}$ & 2762 & $\mathrm{U}$ & VP1 & 95 & Ser & $>8.0$ \\
\hline Mah-KKVP2Y 142 & 1373 & $\mathrm{U}$ & VP2 & 142 & Tyr & 8.0 \\
\hline Mah-KKVP3K 60 & 1944 & A & VP3 & 60 & Lys & 7.0 \\
\hline Mah-KKVP4V 62 & 926 & G & VP4 & 62 & Val & 7.5 \\
\hline
\end{tabular}

gous positions (43 and 95 of VP1, 142 of VP2, and 60 of VP3). Two of these residues, 95 of VP1 and 60 of VP3, were already present in the Sabin strain, and the other two were selected in the PVpiS11 during persistent infection in IMR-32 cells.

To analyze which mutation(s) was responsible for the mouse adaptation phenotype of PVpi170, we assayed the neurovirulence for mouse of PV-1/Mahoney containing each of the five missense mutations independently. These viral mutants were derived from plasmid pKK17. They were designated Mah-KK followed by the name of the modified capsid protein and by the abbreviation and the position of the substituted amino acid, i.e., Mah-KKVP1V ${ }_{43}$, Mah-KKVP1S ${ }_{95}$, Mah-KKVP2 ${ }_{142}$, Mah-KKVP3K 60 , and Mah-KKVP4V 62 (Table 1).

$\mathrm{C} \rightarrow \mathrm{U}$ substitutions were introduced at nucleotides 2607 and 2762 of the VP1 gene of PV-1/Mahoney by site-directed mutagenesis into M13/KK-VP1, using the oligonucleotide primers 5'-GGAAATTCCGGTACTCACCGC-3' and 5'-CC GTGGATAACTCAGCTTCCACC-3', respectively, as described above. The 484-bp NheI-SnaBI fragments from the mutated replicative forms carrying each isolated mutation were used to replace the corresponding fragment in pKK17 to give $\mathrm{pKK}-\mathrm{VP} 1 \mathrm{~V}_{43}$ and $\mathrm{pKK}-\mathrm{VP1S} \mathrm{S}_{95}$, respectively.

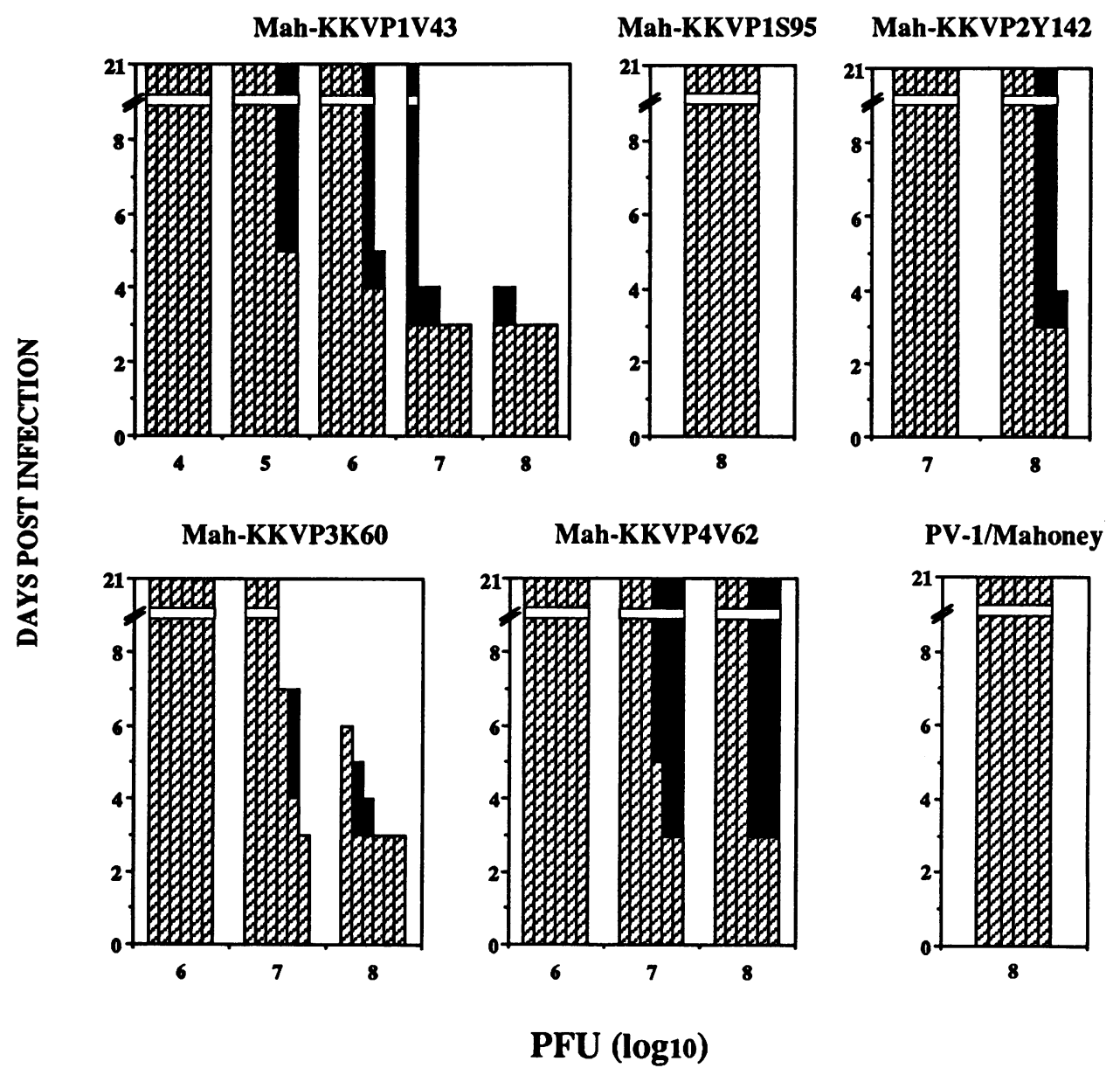

FIG. 2. Neurovirulence tests in mice of PV mutants. The tests were performed as described in the text. Amounts of viruses inoculated per animal are indicated in $\log _{10}$ PFU at the bottom. The heights of the vertical bars represent the life spans of individual mice. Hatched bars and filled bars indicate survivors without any clinical symptoms and with paralysis, respectively. Results of an experiment with mice inoculated with PV-1/Mahoney are also shown. 

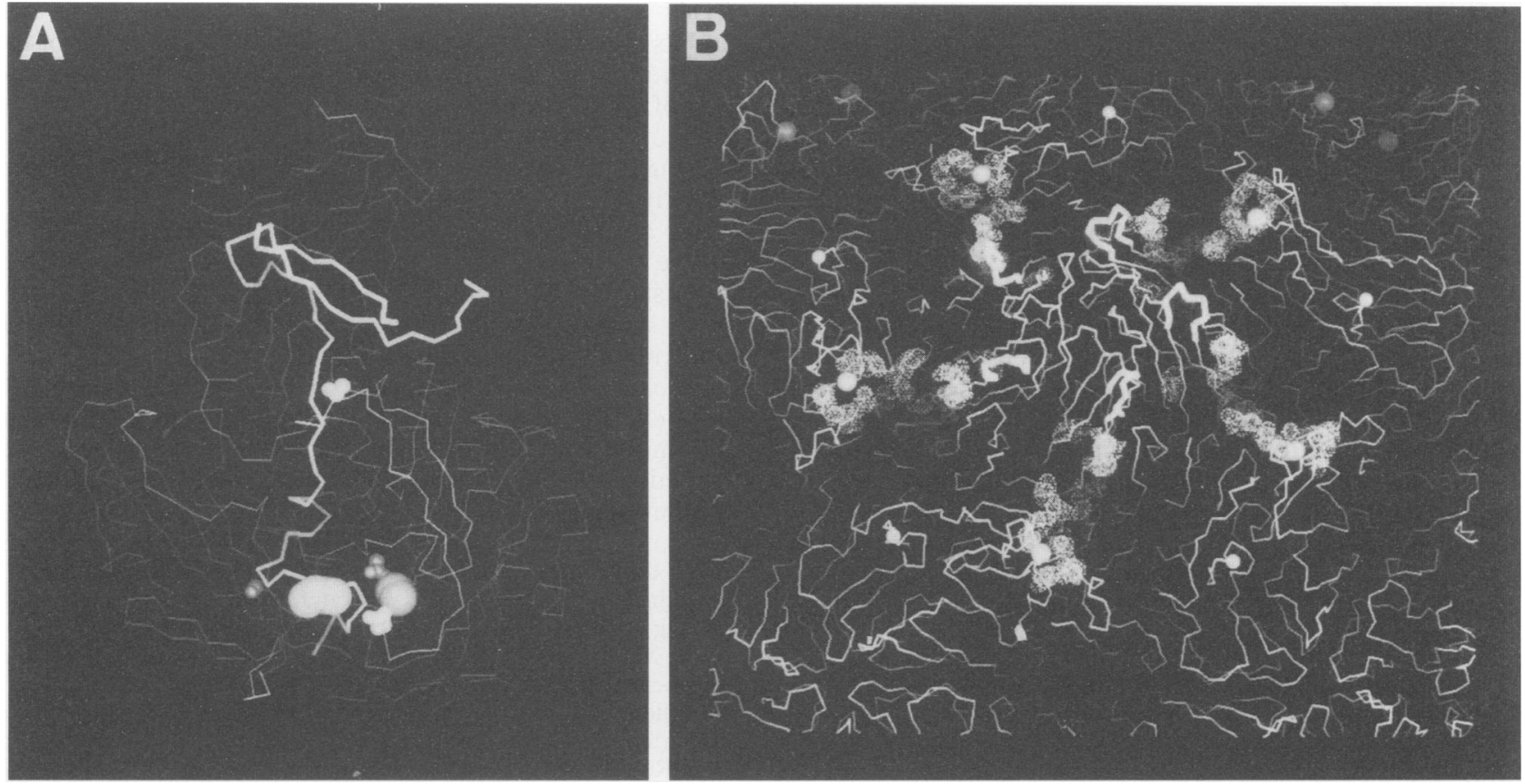

FIG. 3. Locations of the mutations which confer mouse virulence. (A) The mutations on the inner surface of the capsid. The backbone model for a single protomer (VP1, VP2, VP3, and VP4) is shown as viewed from the inside of the particle. The side chains of the residues involved in the two internal mutations identified here (VP4 residue 62 is left of center and VP1 residue 43 is right of center) are indicated by large spheres, and the side chains of the residues involved in the internal mutations previously identified as important in mouse adaptation (residues 22 , 40, and 54 of VP1 and residue 31 of VP2) $(5,18)$ are shown as small spheres. The inner surface of the capsid shell is closest to the viewer, with the fivefold axis located at the top center and the threefold axis located at the bottom center. (B) Locations of mutations on the outer surface of the virus. The view encompasses several protomers in the vicinity of the "canyon" which surrounds the particle fivefold axis. The B-C loops (residues 94 to 102 ) of several fivefold axis-related copies of VP1 are shown as thick lines, the alpha carbons of several symmetry-related copies of VP2 residue 142 are shown as large spheres, and the alpha carbons of several symmetry-related copies of VP3 residue 60 are shown as small spheres. The surfaces corresponding to the symmetry-related footprints of ICAM-1 on rhinovirus 14 (20) are indicated by the stippled dot surfaces. (Figure by R. Grant, Harvard Medical School.)

The substitutions $\mathrm{A} \rightarrow \mathrm{G}$ and $\mathrm{C} \rightarrow \mathrm{U}$ were introduced by site-directed mutagenesis at nucleotides 926 and 1373 of the VP4 and VP2 genes, respectively, of PV-1/Mahoney. The mutations were introduced into the single-strand form of M13/KK-VP2 (5) containing the PV-1/Mahoney cDNA sequence from nucleotide 670 to 1810 , using the oligonucleotide primers 5'-GCTGTTTTTACCAGGACATCC-3' and 5'-GCT GGTGTACATGGTAGTGG-3', respectively. The 648-bp BstBIBsmI fragments from the mutated replicative forms were used to substitute for the corresponding fragment in $\mathrm{pSP} / \mathrm{KK}-5 \mathrm{NC}$ (5). This plasmid carries the 2,991-bp XhoI-SnaBI fragment from pKK17 including the PV-1/Mahoney sequence from nucleotide 1 to 2954. The 2,507-bp XhoI-NheI fragments from the resulting plasmids were inserted in place of their homologs in $\mathrm{pKK} 17$ to give $\mathrm{pKK}-\mathrm{VP} 4 \mathrm{~V}_{62}$ and $\mathrm{pKK}-\mathrm{VP} 2 \mathrm{Y}_{142}$, respectively.

The substitution C-1944 $\rightarrow$ A was introduced by amplifying a fragment corresponding to nucleotides 1924 to 2924 of the cDNA from pKK17 by PCR as described previously (1). In this reaction, the upstream primer corresponding to nucleotides 1924 to 1953 carried a $C \rightarrow$ A substitution at nucleotide position 1,944 and an $A f l$ II site (5'-CCCTTTGACTTAAGTGCCAAA AAAAAGAACACCATG-3'. The 537-bp $A f l$ II-NheI fragment obtained from the amplified mutated fragment was inserted in place of the corresponding fragment into pSP/KK-5NC. The 2,507-bp XhoI-NheI fragment from the resulting plasmid was used to substitute for the corresponding fragment in pKK17 to give $\mathrm{pKK}-\mathrm{VP} 3 \mathrm{~K}_{60}$.
Viruses Mah-KKVP1V ${ }_{43}$, Mah-KKVP1S ${ }_{95}$, Mah-KKVP2 ${ }_{142}$, Mah-KKVP3K 60 , and Mah-KKVP4V $\mathrm{V}_{62}$ were recovered after transfection into Vero cells with purified plasmids $\mathrm{pKKVP1V}_{43}, \mathrm{pKKVP1S}_{95}, \mathrm{pKKVP2}_{142}, \mathrm{pKKVP3K}_{60}$, and pKKVP4V $\mathrm{V}_{62}$, respectively, as previously described (5), and the presence of the mutations in the genome of the viruses was verified.

The neurovirulence of the various PV mutants was then tested by inoculating mice as described above. For each neurovirulent mutant, the sequence of viral RNA recovered from the spinal cord of one paralyzed mouse was verified as described previously (5). Although the neurovirulence of mutated viruses, as assessed from clinical symptoms and number of deaths, differed from one virus to another, all of the mutated viruses except Mah-KKVP1S ${ }_{95}$ were adapted to the mouse central nervous system (Fig. 2 and Table 1). Four new mouse adaptation determinants located in VP1 (residue 43), VP2 (residue 142), VP3 (residue 60), and VP4 (residue 62) were thus identified. Mah-KKVP1V ${ }_{43}$ and Mah-KKVP3K 60 appeared to kill some mice before any paralysis could be observed. This phenomenon was observed at the highest doses of virus and with the most neurovirulent mutants. With Mah-KKVP4V $\mathrm{V}_{62}$, all mice exhibiting paralysis survived until the end of the test (21 days). Mah-KKVP1V 43 , Mah-KKV $\mathrm{P} \mathrm{K}_{60}$, and Mah-KKVP4V 62 were more virulent than PVpi170. This may be due either to some attenuation mutations carried by the genome of PVpi170 or to an antagonist effect of the 
association of the amino acid substitutions in the capsid proteins.

Results mentioned above and comparison of the amino acid changes in capsid proteins of PVpi170 with those of PVpiS11 indicated that PVpiS11 harbors four residues (22 and 43 of VP1, 142 of VP2, and 60 of VP3) that conferred the mousevirulent phenotype to the PV-1/Mahoney strain. Therefore, mouse-neurovirulent determinants can be selected in neuroblastoma cells persistently infected with the attenuated PV-1/ Sabin strain as well as with the wild-type PV-1/Mahoney strain.

Residues 43 of VP1 and 62 of VP4 are located on the inner surface of the capsid in the area containing internal mouse adaptation determinants previously described (residues 22,40 , and 54 of VP1 and 31 of VP2) $(5,10,18)$ (Fig. 3A). This area may be involved in the conformational changes of $\mathrm{PV}$ virions following attachment to the PV receptor required for entry and uncoating $(8,9)$. The mouse adaptation determinants located in this functional area may thus facilitate the conformational changes during early steps of mouse nerve cell infection, allowing PV-1/Mahoney to overcome its host range restriction to mice, as suggested elsewhere $(5,18)$.

Residues 142 of VP2 and 60 of VP3 are located in loops exposed at the surface of the protein shell (10). The first one is located in the E-F loop of VP2, close to antigenic site 2, and the second is carried by the loop preceding the $\beta$ strand $B$ of VP3, a major component of antigenic site 3a. Both of these residues are highly accessible on the surface of the virion and may be directly involved in binding to specific receptors in the mouse central nervous system. Although the nature of interactions between PV and its receptor is not known, a low resolution of a complex between the structurally related rhinovirus 16 and a two-domain fragment of its receptor ICAM-1 has been determined by cryomicroscopy and image reconstruction methods (20). Residues corresponding to the B-C loop of VP1 and residue 142 of VP2 fall either within or at the periphery of the footprint of the receptor on the virus (Fig. 3B). However, the residue analogous to VP3 residue 60 falls well outside the footprint. This may suggest that the nature of the interaction of PV with its receptor differs from the interaction of rhinovirus with its receptor. Alternatively, VP3 residue 60 (as well as VP2 residue 142 and the B-C loop of VP1) may be interacting with an auxiliary protein such as CD44 (see below) in the mouse central nervous system, or it may be playing an as yet undetermined role in events downstream of receptor attachment. In this regard, it is interesting that VP3 residue 60 falls within a very unusual loop structure which is highly conserved among picornavirus structures. To our knowledge, this is the first time that a biological function (outside of being a primary site for immune recognition) has been ascribed to this conserved structure.

Mutations modifying amino acids involved in the early steps of PV infection were selected in persistently infected neuroblastoma cells. It is noteworthy that all the substitutions in the capsid proteins of PVpi170 except one conferred mouse adaptation to PV-1. Moreover, three of these residues are also carried by PVpiS11. Together, the data suggest that mutations would have arisen as a response to the selective pressure of a cellular factor(s) involved in early steps of PV multiplication. As mutations selected in neuroblastoma cells conferred to PV-1 the ability to infect murine neurons, an attractive hypothesis is that the selective factor would be specifically expressed by neuronal cells.

Recently, it has been shown that the lymphocyte homing receptor CD44 either may be a component of the PV receptor site or may interact with the PV receptor through the cytoskeleton or signal transduction pathways (23). The gene encoding
CD44 is expressed in diverse derivatives of the neural crest, and its tissue distribution correlates with the tissue tropism of PV in humans (23). However, it has been shown that expression of CD44 is repressed in neuroblastoma cells (24). The low level of CD44 in these cells might exert a selective pressure leading to PVpi which carry amino acid substitutions involved in early steps.

We are currently investigating the involvement of the new mouse adaptation determinants that we have identified in the early steps of the viral growth cycle and the possible role of these substitutions in the neurovirulent phenotype in transgenic mice expressing the human PV receptor.

(Biohazards associated with the experiments described in this publication have been previously examined by the French Commission de Génie Génétique, and the experiments were carried out in compliance with the rules established by this committee.)

We thank R. Grant for preparation of Fig. 3 and K. Kean for providing plasmid pKK17. We are grateful to F. Delpeyroux for critical reading of the manuscript.

The Ministère de l'Enseignement Supérieur et de la Recherche and the Société de Secours des Amis des Sciences are acknowledged for the fellowships given to N.G. and V.C., respectively. This work was supported by grants from the Direction Scientifique des Applications de la Recherche de l'Institut Pasteur (CCAR grant 27 177) and from the Institut National de la Santé et de la Recherche Médicale (CRE 91 1301 and 91 0107).

\section{REFERENCES}

1. Balanant, J., S. Guillot, A. Candrea, F. Delpeyroux, and R. Crainic. 1991. The natural genomic variability of poliovirus analyzed by a restriction fragment length polymorphism assay. Virology 184:645-654.

2. Borzakian, S., I. Pelletier, V. Calvez, and F. Colbère-Garapin. 1993. Precise missense and silent mutations are fixed in the genomes of poliovirus mutants from persistently infected cells. J. Virol. 67:2914-2917.

3. Calvez, V., I. Pelletier, S. Borzakian, and F. Colbère-Garapin. 1993. Identification of a region of the poliovirus genome involved in persistent infection of HEp-2 cells. J. Virol. 67:4432-4435.

4. Colbère-Garapin, F., C. Christodoulou, R. Crainic, and I. Pelletier. 1989. Persistent poliovirus infection of human neuroblastoma cells. Proc. Natl. Acad. Sci. USA 86:7590-7594.

5. Couderc, T., J. Hogle, H. Le Blay, F. Horaud, and B. Blondel. 1993. Molecular characterization of mouse-virulent poliovirus type 1 Mahoney mutants: involvement of residues of polypeptides VP1 and VP2 located on the inner surface of the capsid protein shell. J. Virol. 67:3808-3817.

6. Fichot, O., and M. Girard. 1990. An improved method for sequencing of RNA templates. Nucleic Acids Res. 18:6162.

7. Filman, D. J., R. Syed, M. Chow, A. J. Macadam, P. D. Minor, and J. M. Hogle. 1989. Structural factors that control conformational transitions and serotypes specificity in type 3 poliovirus. EMBO J. 8:1567-1579.

8. Flore, O., C. E. Fricks, D. J. Filman, and J. M. Hogle. 1990. Conformational changes in poliovirus assembly and cell entry, p. 429-438. In J. M. Hogle (ed.), Seminars in virology. W. B. Saunders Co., London.

9. Fricks, C. E., and J. M. Hogle. 1990. Cell-induced conformational change in poliovirus: externalization of the amino terminus of VP1 is responsible for liposome binding. J. Virol. 64:1934-1945.

10. Hogle, J. M., M. Chow, and D. J. Filman. 1985. Three-dimensional structure of poliovirus at $2.9 \AA$ resolution. Science 229:1358-1365.

11. Kean, K. M., C. Wychowski, H. Kopecka, and M. Girard. 1986. Highly infectious plasmids carrying poliovirus cDNA are capable of replication in transfected simian cells. J. Virol. 59:490-493.

12. Koike, S., H. Horie, I. Ise, A. Okitsu, M. Yoshida, N. Iizuka, K. Takeuchi, T. Takegami, and A. Nomoto. 1990. The poliovirus receptor protein is produced both as membrane-bound and secreted forms. EMBO J. 9:3217-3224. 
13. Koike, S., C. Taya, T. Kurata, S. Abe, I. Ise, H. Yonekawa, and A. Nomoto. 1991. Transgenic mice susceptible to poliovirus. Proc. Natl. Acad. Sci. USA 88:951-955.

14. La Monica, N., J. W. Almond, and V. R. Racaniello. 1987. A mouse model for poliovirus neurovirulence identifies mutations that attenuate the virus for humans. J. Virol. 61:2917-2920.

15. Lonberg-Holm, K. L., L. B. Gosser, and J. J. Kauer. 1975. Early alteration of poliovirus in infected cells and its specific inhibition. J. Gen. Virol. 27:329-342.

16. Martin, A., D. Bénichou, T. Couderc, J. M. Hogle, C. Wychowski, S. van der Werf, and M. Girard. 1991. Use of a type 1/type 2 chimaeric poliovirus to study determinants of poliovirus type 1 neurovirulence in a mouse model. Virology 180:648-658.

17. Mendelsohn, C., E. Wimmer, and V. R. Racaniello. 1989. Cellular receptor for poliovirus: molecular cloning, nucleotide sequence and expression of a new member of the immunoglobulin superfamily. Cell 56:855-865.

18. Moss, E. G., and V. R. Racaniello. 1991. Host range determinants located on the interior of the poliovirus capsid. EMBO J. 10:10671074.
19. Murray, M. G., J. Bradley, X. F. Yang, E. Wimmer, E. G. Moss, and V. R. Racaniello. 1988. Poliovirus host range is determined by a short amino acid sequence in neutralization antigenic site 1 . Science 241:213-215.

20. Olson, N. H., P. R. Kolatkar, M. A. Oliveira, R. H. Cheng, J. M. Greve, A. McClelland, T. S. Baker, and M. G. Rossmann. 1993. Structure of a human rhinovirus complexed with its receptor molecule. Proc. Natl. Acad. Sci. USA 90:507-511.

21. Pelletier, I., T. Couderc, S.Borzakian, E. Wyckof, R. Crainic, E. Ehrenfeld, and F. Colbère-Garapin. 1991. Characterization of persistent poliovirus mutants selected in human neuroblastoma cells. Virology 180:729-737.

22. Ren, R., F. Costantini, E. J. Gorgacz, J. J. Lee, and V. R. Racaniello. 1990. Transgenic mice expressing a human poliovirus receptor: a new model for poliomyelitis. Cell 63:353-362.

23. Shepley, M. A., and V. R. Racaniello. 1994. A monoclonal antibody that blocks poliovirus attachment recognizes the lymphocyte homing receptor CD44. J. Virol. 68:1301-1308.

24. Shtivelman, E., and J. M. Bishop. 1991. Expression of CD44 is repressed in neuroblastoma cells. Mol. Cell. Biol. 11:5446-5453. 